PHYSICAL REVIEW B 84, 104427 (2011)

\title{
Shifted loops and coercivity from field-imprinted high-energy barriers in ferritin and ferrihydrite nanoparticles
}

\author{
N. J. O. Silva* and V. S. Amaral \\ Departamento de Física and CICECO, Universidade de Aveiro, PT-3810-193 Aveiro, Portugal \\ A. Urtizberea, R. Bustamante, A. Millán, and F. Palacio \\ Instituto de Ciencia de Materiales de Aragón, CSIC-Universidad de Zaragoza, Departamento de Fisica de la Materia Condensada, \\ Facultad de Ciencias, ES-50009 Zaragoza, Spain \\ E. Kampert and U. Zeitler \\ Radboud University Nijmegen, Institute for Molecules and Materials, High Field Magnet Laboratory, NL-6525 ED Nijmegen, Netherlands \\ S. de Brion \\ Institut Néel-CNRS and Université Joseph Fourier BP 166, FR-38042 Grenoble cedex 9, France \\ Ò. Iglesias and A. Labarta \\ Departament de Física Fonamental, Universitat de Barcelona and Institut de Nanociència i Nanotecnologia, Universitat de Barcelona, \\ Martí i Franquès 1, ES-08028 Barcelona, Spain
}

(Received 28 October 2010; revised manuscript received 12 August 2011; published 15 September 2011)

\begin{abstract}
We show that the coercive field in ferritin and ferrihydrite depends on the maximum magnetic field in a hysteresis loop and that coercivity and loop shifts depend both on the maximum and cooling fields. In the case of ferritin, we show that the time dependence of the magnetization also depends on the maximum and previous cooling fields. This behavior is associated to changes in the intraparticle energy barriers imprinted by these fields. Accordingly, the dependence of the coercive and loop-shift fields with the maximum field in ferritin and ferrihydrite can be described within the frame of a uniform-rotation model considering a dependence of the energy barrier with the maximum and the cooling fields.
\end{abstract}

DOI: $10.1103 /$ PhysRevB.84.104427

PACS number(s): 75.30.Cr, 75.50.Ee, 75.60.Ej, 75.50.Tt

\section{INTRODUCTION}

The magnetic properties of ferritin have been extensively studied in the last decade due to their puzzling features, such as the existence of a maximum in the magnetization derivative at zero field, ${ }^{1,2}$ a nonmonotonic field dependence of the magnetic viscosity, ${ }^{1-3}$ and a decrease of the antiferromagnetic susceptibility with temperature below the Néel temperature when considered at low fields. ${ }^{4,5}$ Many of these studies were performed to enlighten the possible existence of quantum tunneling in ferritin in the Kelvin range. Less attention has been paid to the magnetic hysteresis, although it was termed "anomalous" in an early report due to the large coercivity $(\sim 1800$ Oe at $5 \mathrm{~K})$, irreversibility up to relatively high fields ( $\sim 35 \mathrm{kOe}$ ), and loop displacement found after field cooling. ${ }^{4}$ Ferritin consists of a hollow spherical shell composed of 24 protein subunits surrounding a ferrihydritelike core. The diameter of the cavity is of the order of 7-8 nm and the average size of the core of horse spleen ferritin is $5 \mathrm{~nm} .{ }^{6}$ The ferritin magnetic core orders antiferromagnetically and has both compensated and uncompensated spins, resulting in a net magnetic moment of about 100 Bohr magnetons $\mu_{B}$ per particle. $^{7}$

Ferritin belongs to a group of nanoparticles where loop displacements are found, but where, strictly speaking, there is neither a ferromagnetic (FM) material coupled to an antiferromagnetic (AF) one nor a cooling across a Néel transition temperature. The cooling is normally performed across the blocking temperature of the nanoparticles, i.e., across the temperature below which the magnetic moment of the "average" nanoparticle is not able to fluctuate across the anisotropy barrier within the characteristic time of the measurement, which in ferritin and for dc measurements is about $20 \mathrm{~K}$. In these systems, the origin of the loop shift is not clearly established. In AF nanoparticles, the loop shift has been often interpreted as an exchange bias between the $\mathrm{AF}$ core and the uncompensated spins of the spin-glass surface. In the case of FM and ferrimagnetic nanoparticles, the loop shift is thought to arise due to exchange between the spin-glass spins and the FM core. ${ }^{8,9}$ Other studies attribute the loop shift to the existence of a minor hysteresis loop. ${ }^{10}$ In fact, as highlighted in Refs. 8 and 9, many of these systems show high-field irreversibility and nonsaturating hysteresis loops, raising the question of the influence of the minor loops on the exchange field and, eventually, the question of the existence of a real exchange bias.

Here, we report a study on the coercive field and loop shifts in ferritin at low temperature, obtained in magnetization cycles recorded for different maximum fields up to $30 \times 10^{4} \mathrm{Oe}$, and after cooling under the influence of fields with different intensities. This study is also extended to ferrihydrite nanoparticles, which are similar to the ferritin magnetic cores, and complemented by the measurement of the time dependence of the magnetization near zero field. 


\section{BACKGROUND}

Within the framework of the uniform rotation models developed by Stoner-Wohlfarth and Néel-Brown, ${ }^{11,12}$ reversal of magnetization over an energy barrier $E$ separating two minima is a coherent process, which can occur at $T=0 \mathrm{~K}$ when the barrier is lowered down to zero by applying a magnetic field in the opposite direction of the particle's magnetization, ${ }^{13}$ or it can occur by thermal activation when the thermal energy $k_{B} T$ becomes comparable to $E$ and, thus, the characteristic reversal time $\tau$ becomes comparable to the characteristic measurement time $\tau_{m} .{ }^{11}$ These quantities are related by the Arrhenius law

$$
\tau=\tau_{0} \exp \left(\frac{E}{k_{B} T}\right),
$$

where $\tau_{0}$ is the inverse of an attempt frequency, supposed constant for simplicity in many situations. The energy barrier is field dependent and it can be written as

$$
E(H)=E_{0}\left(1-\frac{H}{H_{0}}\right)^{\alpha}
$$

with $\alpha=2$ for systems with uniaxial anisotropy and easy axes parallel to the applied field, where $E_{0}$ is the energy barrier at zero field and $H_{0}$ is the switching field at zero temperature. In ferromagnetic materials, $H_{0}=2 K / M_{S}$ and $E_{0}=K V$, while in general

$$
E_{0}=K V^{p}
$$

where $V$ is the particle volume, $K$ is the anisotropy constant, $M_{S}$ is the saturation magnetization, and $p$ is an exponent equal to $1 / 2$ in the case of antiferromagnetic ferrihydrite nanoparticles. ${ }^{14}$ In the framework of the Néel model and for a random distribution of anisotropy axes, $\alpha=4 / 3 .{ }^{15}$ In general, simulations and experimental results show that $\alpha$ depends on the anisotropy, distribution of particle sizes, and on interparticle interactions. ${ }^{16,17}$

According to Eq. (2), magnetization reversal occurs at the coercive field $H_{C}$, when the energy barrier $E\left(H_{C}\right)$ becomes small enough to be overcome at the given $T$ and measuring time $\tau$ :

$$
H_{C}=H_{0}\left[1-\left(\frac{E\left(H_{C}\right)}{E_{0}}\right)^{1 / \alpha}\right],
$$

where we have just rewritten Eq. (2). The dependence of $H_{C}$ on temperature, nanoparticle volume, and characteristic measurement time can then be obtained by using Eq. (1) for $E\left(H_{C}\right)$ in the previous equation, as shown in Refs. 18 and 19. In particular, for the volume dependence of $H_{C}$ at constant temperature, one has

$$
H_{C}(V)=H_{0}\left[1-\left(V_{B} / V\right)^{(p / \alpha)}\right],
$$

where $V_{B}$ is the blocking volume, i.e., the volume above which $E_{0}$ can not be crossed within $\tau_{m}$ in a system with anisotropy $K$ and at a temperature $T$. A more refined expression for $H_{C}(T)$ can be obtained by considering the temperature dependence of $K$ and $M_{S} .^{20}$

\section{EXPERIMENT}

Horse spleen ferritin samples used in these experiments were obtained from Sigma Chemical Company and prepared in powder samples by evaporation of the solvent at room temperature.

The synthesis of the ferrihydrite nanoparticles in the organic-inorganic matrix (termed di-ureasil) has been described elsewhere. ${ }^{21}$ The particles are precipitated by thermal treatment at $80{ }^{\circ} \mathrm{C}$, after the incorporation of iron nitrate in the matrix. The sample here studied has an iron concentration of $2.1 \%$ in weight and the particles have a diameter distribution that can be described by a lognormal function

$$
f(D)=\frac{1}{D s_{D} \sqrt{2 \pi}} \exp \left[-\frac{\left[\log \left(D / n_{D}\right)\right]^{2}}{2 s_{D}^{2}}\right]
$$

with $n_{D}=4.7 \pm 0.2 \mathrm{~nm}$ and standard deviation of the natural logarithm of the diameter $s_{D}=0.43 \pm 0.05 .^{22}$

For ferritin, magnetization was measured as a function of field up to different maximum fields $H_{\text {max }}$ (in the $0.5 \times 10^{4}$ to $30 \times 10^{4}$ Oe range) and after cooling from $100 \mathrm{~K}$ down to low temperature $(3.2$ and $4.2 \mathrm{~K})$ in zero-field cooling (ZFC) and field-cooling (FC) procedures using different cooling fields $H_{\text {cool }}$. These measurements were performed in a PPMS system (Quantum Design) with a vibrating sample magnetometer (VSM) option, and in a Bitter magnet with a VSM (HFML facility, Nijmegen). In the latter, the modulus of the magnetization was measured and magnetization curves were reconstructed by using the proper signal. Near zero, this procedure is not perfect (since noise is always additive) and a small kink around zero field appears (see Fig. 1).

At the characteristic time of measurement, irreversibility phenomena vanish for $T>40 \mathrm{~K}$ and magnetization curves taken at $3.2 \mathrm{~K}$ after cooling with $H_{\text {cool }}=2 \times 10^{4}$ Oe from temperatures between 50 and $300 \mathrm{~K}$ are similar. The magnetization curves are also independent on the cooling rate (cooling from $100 \mathrm{~K}$ ) in the 0.5 to $5 \mathrm{~K} / \mathrm{min}$ range. In addition, magnetization was measured as a function of time during about $1000 \mathrm{~s}[M(t)]$ after cooling from $100 \mathrm{~K}$ down to $4.5 \mathrm{~K}$ in $\mathrm{ZFC}$ and FC with $H_{\text {cool }}=0.5 \times 10^{4}$ Oe procedures. For each cooling procedure and at low temperature $(4.5 \mathrm{~K})$, we have followed two different measurement protocols: (i) applied different $H_{\max }$, removed the field down to a value near zero $(50 \mathrm{Oe})$, and measured $M(t)$ and (ii) applied different $H_{\max }$, then reversed the field to $-H_{\max }$, removed it down to $-50 \mathrm{Oe}$, and measured $M(t)$.

For the ferrihydrite nanoparticles grown in the organicinorganic hybrid matrix, magnetization was measured as a function of field up to different maximum fields $H_{\max }$ (in the $2 \times 10^{4}$ to $20 \times 10^{4}$ Oe range) and after cooling from $100 \mathrm{~K}$ down to $3.2 \mathrm{~K}$ in $\mathrm{FC}$ procedure using $H_{\text {cool }}=2 \times 10^{4} \mathrm{Oe}$, in a Bitter magnet with an extraction magnetometer (GHMFL facility, Grenoble).

\section{RESULTS AND DISCUSSION}

\section{A. Effect of $\boldsymbol{H}_{\max }$ and $\boldsymbol{H}_{\text {cool }}$ in the magnetization loops}

The magnetization loops of ferritin obtained at low temperature are analogous to those previously reported, ${ }^{1,4,23}$ with nonsaturation, high-field irreversibility, and a maximum in the magnetization derivative at zero field. The ZFC cycles 


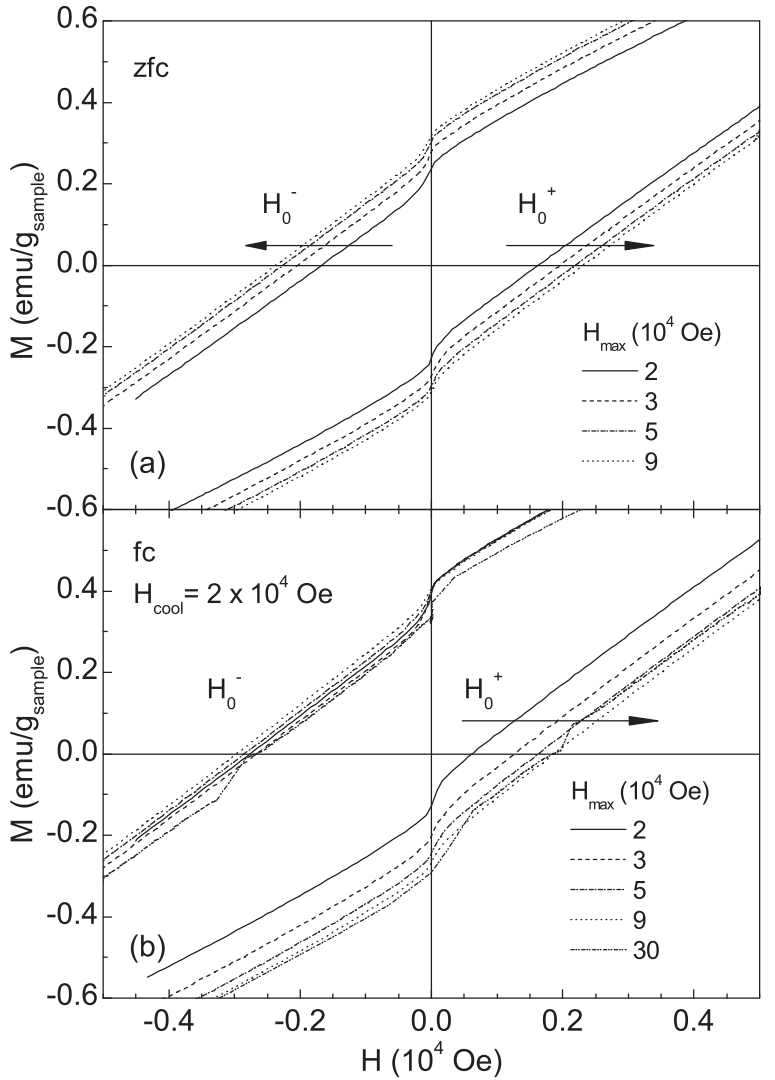

FIG. 1. Detail of magnetization loops for ferritin obtained at $3.2 \mathrm{~K}$ ( $4.2 \mathrm{~K}$ in the case of $H_{\max }=30 \times 10^{4} \mathrm{Oe}$ ) after ZFC (a) and FC with $H_{\text {cool }}=2 \times 10^{4}$ Oe (b) measured for different $H_{\text {max }} . H_{0}^{-}$and $H_{0}^{+}$ correspond to the field values at which magnetization crosses zero in the decreasing and increasing field branches of the hysteresis loop, respectively.

are symmetric and increasingly broader as $H_{\max }$ increases [Fig. 1(a)]. When the sample is cooled in the presence of a field $H_{\text {cool }}$, the magnetization curves are shifted in the $H$ axis [Fig. 1(b)], being also increasingly broader as $H_{\max }$ increases up to fields of the order of $10 \times 10^{4} \mathrm{Oe}$. Another interesting observation is that the differences in decreasing-field branches of the loops obtained after a FC procedure for different $H_{\max }$ are less significant than those obtained after a ZFC procedure, while in increasing-field branches, the differences are more significant after FC than after ZFC procedure. Similar results are found for ferrihydrite nanoparticles.

The horizontal shift of the hysteresis loops in the coolingfield direction is similar to that previously found in ferritin, ${ }^{4}$ ferrihydrite, ${ }^{24}$ and other magnetic nanoparticles. ${ }^{9}$ In the case of $\mathrm{AF} \mathrm{NiO}$ nanoparticles, this loop shift was associated to surface anisotropy and multisublattice states, with the latter being associated to a variety of reversal paths. ${ }^{25}$ Surface anisotropy arises due to the breaking of the crystal-field symmetry at the boundary of the nanoparticle. Two models have been considered: one where the easy axis is transverse to the boundary, and another where the local easy axis depends on the site "defect" (Néel surface anisotropy model). ${ }^{26}$ The loop shift in AF nanoparticles is also interpreted in terms of an exchange bias between antiferromagnetic and uncompensated
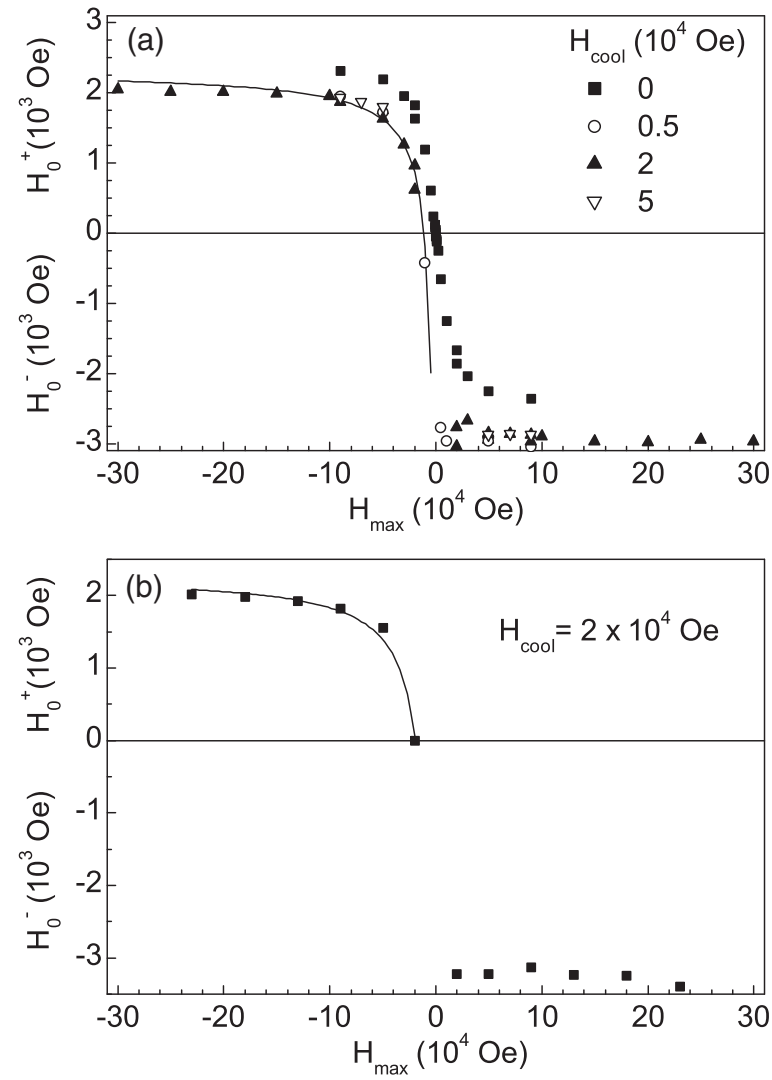

FIG. 2. Fields at which magnetization crosses zero $\left(H_{0}^{-}\right.$and $\left.H_{0}^{+}\right)$ for different cooling fields $H_{\text {cool }}$ as a function of the maximum field $H_{\max }$, for ferritin (a) and ferrihydrite nanoparticles (b). Lines represent fit to Eq. (10).

moments, although no transition temperature is crossed but rather a blocking temperature.

The effect of $H_{\max }$ and $H_{\text {cool }}$ on the field values at which magnetization crosses zero in the decreasing and increasing field branches of the hysteresis loop (termed $\mathrm{H}_{0}^{-}$and $\mathrm{H}_{0}^{+}$, respectively) can be observed in Fig. 2. For $H_{\text {cool }} \neq 0, H_{0}^{-}$has a smaller variation with $H_{\max }$ compared to that of $H_{0}^{+}$, while for $H_{\text {cool }}=0, H_{0}^{-}$and $H_{0}^{+}$have symmetric variations.

From Fig. 2, it is also clear that the effect of $H_{\max }$ on $H_{0}^{+}$is more important than the effect of $H_{\text {cool }}$. In Fig. 3, we show the dependence of $H_{0}^{+}, H_{0}^{-}$, the coercive field $H_{C}=$ $\left(H_{0}^{+}-H_{0}^{-}\right) / 2$, and the loop shift $H_{S}=-\left(H_{0}^{+}+H_{0}^{-}\right) / 2$ on the cooling field $H_{\text {cool }}$ for the highest maximum applied field $H_{\max }=30 \times 10^{4}$ Oe. With increasing $H_{\text {cool }}, H_{0}^{+}$increases approaching the ZFC value for $\mathrm{H}_{\text {cool }} \gtrsim 10^{4} \mathrm{Oe}$, whereas $\left|H_{0}^{-}\right|$ values slightly decrease and are always higher in modulus than the value for ZFC. Interestingly, the larger departures of $H_{0}^{-}$ and $H_{0}^{+}$from the ZFC value occur for lower $H_{\text {cool }}$. As a result, $H_{C}$ is almost independent on $H_{\text {cool }}$, being higher than the ZFC value, while the loop shift $H_{S}$ has a small decrease with $H_{\text {cool }}$.

\section{B. Effect of $\boldsymbol{H}_{\max }$ and $\boldsymbol{H}_{\text {cool }}$ in $\boldsymbol{M ( t )}$}

To have a better insight on the changes occurring near $H_{0}^{-}$ and $H_{0}^{+}$induced by the application of different $H_{\max }$ and $H_{\text {cool }}$, we have performed measurements of $M(t)$ near zero field, after ZFC and FC under $H_{\text {cool }}=0.5 \times 10^{4}$ Oe as described in 


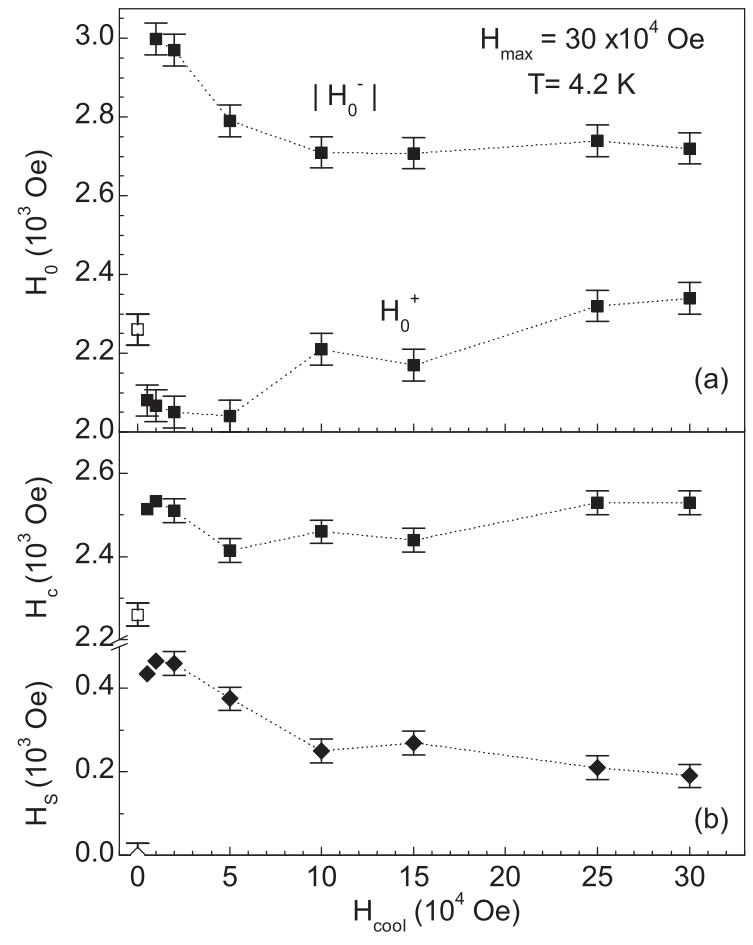

FIG. 3. Dependence of the fields at which magnetization crosses zero $\left(H_{0}^{-}\right.$and $\left.H_{0}^{+}\right)(\mathrm{a}), H_{C}$ and $H_{S}$ (b) on the cooling field $H_{\text {cool }}$ for a maximum field $H_{\max }=30 \times 10^{4} \mathrm{Oe}$, for ferritin. The ZFC values are shown in open symbols (which in the case of $\mathrm{H}_{0}^{-}$and $\mathrm{H}_{0}^{+}$corresponds to the same value). Lines connecting the $\mathrm{FC}$ values are eye guides.

Sec. III. With these measurements, we aim at demonstrating that the changes in the hysteresis loops are related to changes in the energy barriers to magnetization reversal induced by $H_{\max }$ and $H_{\text {cool }}$.

Before discussing the results of the magnetic relaxation measurements presented in Fig. 4(c), we will start by analyzing the magnetic state of the samples attained after the protocols previous to the relaxation measurements. First, we plot in Fig. 4(a) the values of magnetization obtained at $H_{\max }$ $\left(M @ H_{\max }\right)$ after ZFC and FC procedures. As it can be observed, the magnetization values obtained after a FC are always higher than those attained after a ZFC process. Second, the magnetization values obtained right after the field is decreased down to $50 \mathrm{Oe}(-50 \mathrm{Oe})\left[M\left(t_{0}\right)\right]$ after FC and ZFC procedures are plotted in Fig. 4(b). Comparison with results in the previous panel shows that the difference between the ZFC and FC values of $M @ H_{\max }$ (full squares) is always smaller than the differences between the ZFC and FC values of $M\left(t_{0}\right)$ (open circles). In fact, at the highest applied $H_{\max }=$ $5 \times 10^{4}$ Oe, FC and ZFC values of $M @ H_{\max }$ are identical, while the corresponding values for $M\left(t_{0}\right)$ become substantially different. The differences after FC and ZFC procedures that appear in $M\left(t_{0}\right)$ after decreasing the field down to \pm 50 Oe are an indication of the different energy barriers that each particle magnetic moment has been able to cross. The higher $M\left(t_{0}\right)$ values measured after $\mathrm{FC}$ hint at the appearance of energy barriers imprinted after FC that are higher than after the ZFC process. Moreover, the fact that $M @ H_{\max }$ values become the same after measuring at the highest $H_{\max }=5 \times 10^{4}$ Oe [see Fig. 4(a)] indicates that the differences in $M\left(t_{0}\right)$ appearing after

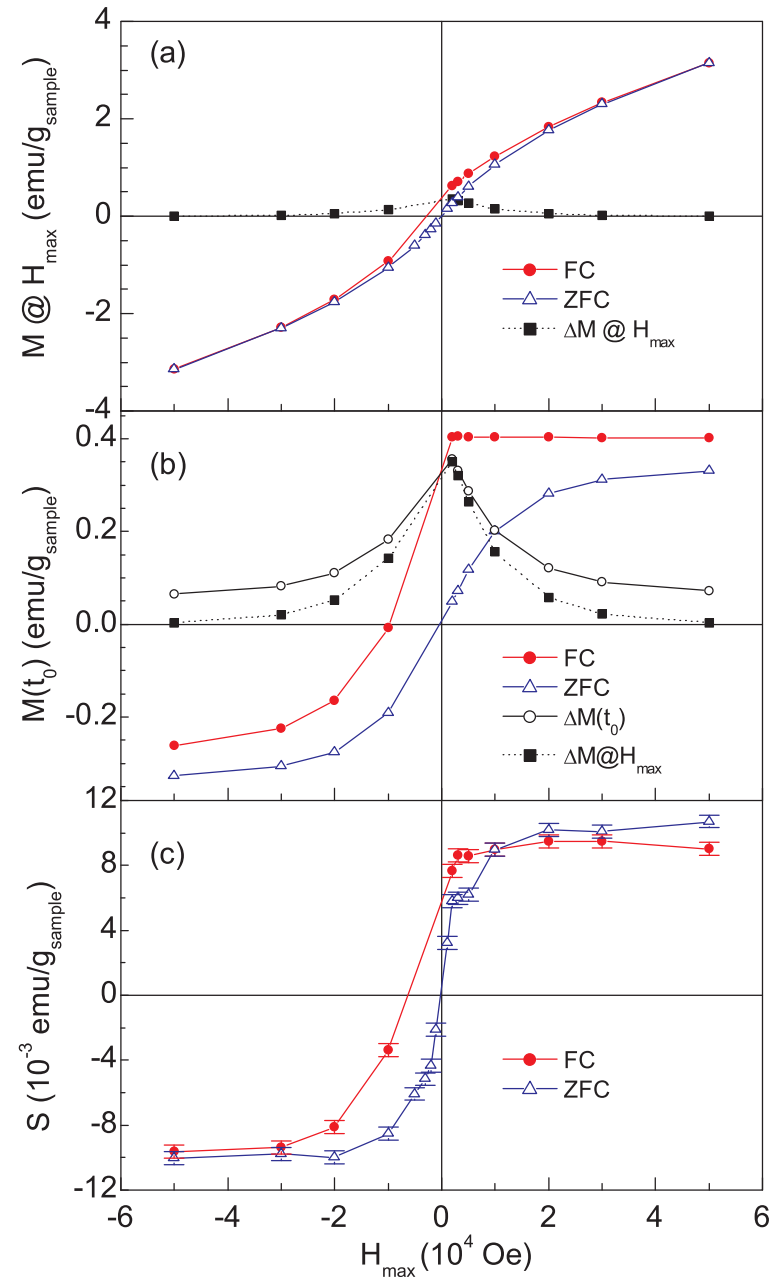

FIG. 4. (Color online) (a) Magnetization measured at $H_{\max }$ $\left(-H_{\max }\right)$ before removing the field down to $50(-50)$ Oe $\left(M @ H_{\max }\right)$ obtained after ZFC and FC with $H_{\text {cool }}=0.5 \times 10^{4} \mathrm{Oe}$, and difference between $M @ H_{\max }$ after FC and ZFC $\Delta M @ H_{\max }$; (b) first value of the remanent magnetization measured at $50(-50)$ Oe $\left[M\left(t_{0}\right)\right]$ obtained after ZFC and FC with $H_{\text {cool }}=0.5 \times 10^{4} \mathrm{Oe}$ and difference between the FC and ZFC values; (c) magnetic viscosity $S$ measured at $50(-50)$ Oe after a previous $H_{\max }\left(-H_{\max }\right)$. Measurements were performed in ferritin.

both procedures can not be attributed to an increase of the net magnetic moment of the individual particles induced at $H_{\max }$ after the FC process. Finally, it is also interesting to note the constancy of $M\left(t_{0}\right)$ observed for positive $H_{\max }$ after FC [filled circles in Fig. 4(b)], which shows that the fraction of magnetic moments that reverse after reduction of the field is almost independent of the maximum applied field and reinforces the two points commented previously. In contrast, when $H_{\max }$ is applied in a direction opposite to $H_{\text {cool }}$ [points with negative abscissas in panels (a) and (b) of Fig. 4], there is a progressive increase of $M\left(t_{0}\right)$ with increasing $H_{\max }$ for both ZFC and FC procedures, while $M @ H_{\max }$ values are essentially the same in the two cases. Again, this shows that the changes in $M\left(t_{0}\right)$ can not be attributed to an increase of the uncompensated moment of the particles but rather to the fact that, when arriving near zero field, different fractions of magnetic moments are able to cross the energy barriers at $t_{0}$ depending on $H_{\max }$ and $H_{\text {cool }}$, an 
indication that the effective energy barriers felt by the particles near zero field are modified by $H_{\text {max }}$. In a simple picture, a negative $H_{\max }$ has the effect of erasing the barriers imprinted by the positive $H_{\text {cool }}$, with the system being closer to the ZFC configuration as the intensity of $H_{\max }$ increases.

In what follows, we will analyze the results of the relaxation measurements following the two above-mentioned protocols. As previously found in ferritin, ${ }^{1,3} M(t)$ displays a quasilinear dependence on $\ln (t)$ at intermediate times within the studied $t$ range (up to $1000 \mathrm{~s}$ ) and can be fitted to the following expression:

$$
M(t) \approx M_{0}^{\prime}-S \ln (t),
$$

where $M_{0}^{\prime}$ is related to the initial magnetization and $S$ is the so-called coefficient of magnetic viscosity. Equation (7) is particularly useful in situations where $\tau_{0}$ is not known. ${ }^{27}$ This equation can be derived from a general expression for the time dependence of the magnetization of an ensemble of nanoparticles with distribution of energy barriers $f(E)$, after field removal

$$
M(t)=\int_{0}^{\infty} M_{0}(E) \exp [-t / \tau(E)] f(E) d E,
$$

where $M_{0}(E)$ is the initial magnetization of a particle with energy barrier $E$. It can also be shown that $S$ is proportional to $E f(E)$ and, therefore, $S$ is an appropriate quantity to observe changes in the energy barriers. ${ }^{27,28}$ However, the direction of this change is not directly given by $S$ since, in principle, $f(E)$ is a nonmonotonous function. A distribution of energy barriers results directly from a distribution of volumes, according to the relation $f(E)=g(V)(d V / d E)$, where $g(V)$ is the volume distribution. Other sources of a distribution of energy barriers are a distribution of shapes, the existence of nanoparticles with the same size but with different degrees of crystallinity and different oxygen and water content.

This equation assumes that the magnetization decay of a nanoparticle ensemble is due to the switching of the nanoparticles magnetic moments as a consequence of energy barrier crossing when, for a given $T$ and $H$, the Arrhenius relaxation time $\tau$ is of the order of the measurement time $\tau_{m}$.

The $H_{\max }$ dependence of the viscosity coefficient $S$ as obtained from fits of the linear part of the relaxation curves to Eq. (7) is reported in Fig. 4(c). We observe that, for positive $H_{\text {max }}$ and for the FC case, $S$ remains essentially constant with increasing $H_{\max }$. This means that $H_{\text {cool }}$ imprints energy barriers for reversal into the FC direction, which is not substantially changed by a positive applied $H_{\max }$. However, in the ZFC case, the relaxation rate increases with $H_{\max }$, showing that $H_{\text {max }}$ changes the energy barriers in this case. For negative $H_{\text {max }}$ (applied contrary to the $H_{\text {cool }}$ direction), however, the energy barriers are shifted. In summary, the general behavior of $S$ is similar to that of $M\left(t_{0}\right)$, reinforcing the interpretation of the effects of $H_{\text {cool }}$ and $H_{\max }$ in terms of energy barriers. Since in ferritin $^{29,30}$ and in the ferrihydrite nanoparticles studied ${ }^{14}$ here interparticle interactions are negligible, the proposed changes in the energy barriers are most probably associated to intraparticle phenomena, as discussed below.

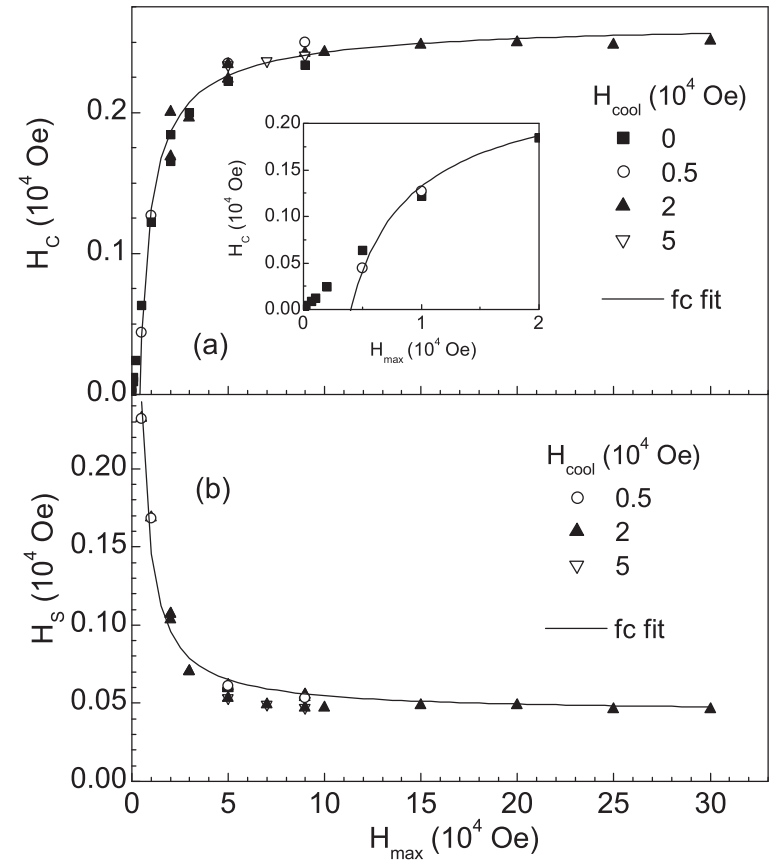

FIG. 5. Dependence of the (a) coercive field $H_{C}$ and (b) loop shift $H_{S}$ on the maximum field $H_{\max }$ in ferritin, for different cooling fields $H_{\text {cool }}$. Lines in panels (a) and (b) represent fits to Eqs. (11) and (12), respectively. Inset shows zoom over the low $H_{\max }$ region.

\section{Effect of $\boldsymbol{H}_{\max }$ on the energy barriers}

In Fig. 5, we present the dependence of $H_{C}$ and $H_{S}$ with $H_{\max }$ for hysteresis loops measured after ZFC (squares) and after cooling in different $H_{\text {cool }}$. The first point to notice is that, in the FC case, the loop shift $H_{S}$ first rapidly decreases for low $H_{\text {max }}$ but, for fields higher than $10 \times 10^{4} \mathrm{Oe}$, it saturates to a value around 500 Oe even for extraordinarily high values of $H_{\max }=30 \times 10^{4}$ Oe. This behavior is somewhat unexpected since, for the usually observed loop shifts due to minor loops, the shift tends to zero for sufficiently high fields.

Second, the constancy of $H_{C}$ obtained at high fields in the FC case is an indication of the existence of high-energy barriers imprinted by $H_{\text {cool }}$, which can not be surmounted even by applying a $H_{\max }$ of $30 \times 10^{4}$ Oe field in the direction opposite to $H_{\text {cool }}$. Both observations (together with the $H_{0}^{+}$variation already presented in Fig. 2) indicate an initial evolution of the minor loops due to crossing of smallest energy barriers. After this first stage, the variations are smoothed by the higherenergy barriers imprinted by $H_{\text {cool }}$. According to the behavior of $H_{0}^{+}, H_{0}^{-}$, and $M\left(t_{0}\right)$, it is clear that $H_{\text {cool }}$ increases the energy barriers in the field direction $\left(E_{0}^{-}\right)$and decreases barriers in the opposite direction $\left(E_{0}^{+}\right)$. Since the highest barriers are not overcome, the symmetric situation $E_{0}^{+}=E_{0}^{-}$can not be recovered and, thus, $H_{S}$ is always different from zero.

A quantitative description of these phenomena can be given within the framework of the uniform-rotation model in terms of the influence of $H_{\max }$ on the energy barriers near zero field $E_{0}$. First, let us notice that the dependence of $H_{C}$ on $H_{\max }$ is similar to the dependence of $H_{C}$ on $V$ usually found in nanoparticle systems [see Eq. (5)]. ${ }^{15}$ Taking into account this resemblance, and with the aim to propose an expression for $H_{C}\left(H_{\max }\right)$, which properly describes the measured data, we 
will assume that $H_{\max }$ influences the zero-field energy barriers in a way similar to the particle volume [Eq. (3)]

$$
E_{0} \propto H_{\max }^{\gamma},
$$

where $\gamma$ is a power-law exponent that controls the way $H_{C}$ approaches its limiting value for high $H_{\max }$, such that a higher $\gamma$ is associated to a faster approach to saturation. This power-law dependence condensates different possible mechanisms for the influence of $H_{\max }$ on intrinsic energy barriers: either a change in the exponent $p$ in Eq. (3) or an irreversible increase of the anisotropy constant $K$. The first possibility seems to be ruled out since, as reported in the preceding section, $H_{\max }$ does not seem to affect the net magnetic moment of the particles. Therefore, the influence of $H_{\text {max }}$ can be thought of mostly as an effect on $K$ associated to an increase of the local (intraparticles) energy barriers. Since these are macroscopically average measurements, it is difficult to access the "microscopic" origin for this effect on $K$. Anyway, this can be understood considering that the system has multiple configurations with associated energies such that $H_{\text {max }}$ and $H_{\text {cool }}$ select or imprint a set of these configurations restricting the relaxation of the moments.

Based on Eq. (4), the relation between $H_{0}^{+}$and $H_{\max }$ can be expressed as

$$
H_{0}^{+}\left(H_{\max }\right)=H_{0}^{+}(\infty)\left[1-\left(\frac{H_{B}^{+}}{H_{\max }}\right)^{\beta_{+}}\right],
$$

where $\beta_{+}=\alpha / \gamma, H_{0}^{+}(\infty)$ is redefined as $H_{0}^{+}$for infinite $H_{\text {max }}$, and $H_{B}^{+}$is defined as the field at which $H_{0}^{+}$is zero. The relation between $E_{0}$ and $H_{B}^{+}$is that expressed in Eq. (9) since $H_{B}^{+}$is a particular case of an $H_{\max }$. When comparing to experimental data, care must be taken and the FC and ZFC cases must be distinguished. In the FC case, $H_{0}^{+}$can be negative and $H_{B}^{+}$is the field at which $H_{0}^{+}$crosses zero (Fig. 2). In the ZFC case, $H_{B}^{+}$is defined as the field at which the behavior of $H_{0}^{+}$at high $H_{\text {max }}$ extrapolates to zero since, in practice, the experimental $H_{0}^{+}$values are not zero at $H_{\max } \leqq H_{B}^{+}$.

Equation (10) can successfully describe the $H_{0}^{+}\left(H_{\max }\right)$ data shown in Fig. 2, with $H_{0}^{+}(\infty)=0.23 \times 10^{4} \mathrm{Oe}, H_{B}^{+}=$ $-1.1 \times 10^{4} \mathrm{Oe}$, and $\beta_{+}=0.8$ for the $\mathrm{FC}$ data of ferritin. In the case of ferrihydrite $H_{0}^{+}(\infty)=0.20 \times 10^{4} \mathrm{Oe}, H_{B}^{+}=$ $-2.0 \times 10^{4} \mathrm{Oe}, \beta_{+}=1.5$. Considering $\alpha=4 / 3, \gamma \approx 1$ in ferritin, and $\gamma \approx 2$ in ferrihydrite, i.e., approximately a linear and quadratic relation between $E_{0}$ and $H_{\max }$. The differences here found for $\gamma$ are associated to the fact that, in ferrihydrite, a smaller $H_{\max }$ is enough to approach $H_{0}^{+}$to saturation. Again, the "microscopic" origin for this mechanism is not clear.

For ZFC data, both $H_{0}^{+}\left(H_{\max }\right)$ and $H_{0}^{-}\left(H_{\max }\right)$ are well described by Eq. (10) for $\left|H_{\max }\right|>\left|H_{B}^{+}\right|$, while for $\mathrm{FC}$, $H_{0}^{-}\left(H_{\max }\right)$ is approximately constant. Accordingly, the generalization to the $H_{\max }$ dependence of the coercive field for $\mathrm{ZFC}$ and FC procedures ( $H_{\mathrm{CZFC}}$ and $H_{\mathrm{CFC}}$, respectively) and of $H_{S}$ is straightforward:

$$
\begin{gathered}
H_{\mathrm{CZFC}}\left(H_{\max }\right)=H_{0}^{+}(\infty)\left[1-\left(\frac{H_{B}^{+}}{H_{\max }}\right)^{\beta_{+}}\right], \\
H_{\mathrm{CFC}}\left(H_{\max }\right)=-\frac{1}{2} H_{0}^{-}+\frac{1}{2} H_{0}^{+}(\infty)\left[1-\left(\frac{H_{B}^{+}}{H_{\max }}\right)^{\beta_{+}}\right],
\end{gathered}
$$

and

$$
H_{S}\left(H_{\max }\right)=-\frac{1}{2} H_{0}^{-}-\frac{1}{2} H_{0}^{+}(\infty)\left[1-\left(\frac{H_{B}^{+}}{H_{\max }}\right)^{\beta_{+}}\right],
$$

where $H_{0}^{-}$is constant.

As expected from the agreement between the $H_{0}^{+}\left(H_{\max }\right)$ data and Eq. (10), Eq. (11) can also be successfully used to describe the $H_{\mathrm{CZFC}}\left(H_{\max }\right)$ and $H_{\mathrm{CFC}}\left(H_{\max }\right)$ data of ferritin in the $0.5 \times 10^{4}<H_{\max }<30 \times 10^{4}$ Oe range, as shown in Fig. 5(a), with $H_{0}^{+}(\infty)=0.24 \times 10^{4}$ Oe and $H_{B}^{+}=0.9 \times 10^{4}$ Oe for the ZFC data, and with the previous $H_{0}^{+}\left(H_{\max }\right)$ parameters and $H_{0}^{-}=-0.297 \times 10^{4}$ Oe for the FC data. For low fields $\left(H_{\max }<0.5 \times 10^{4} \mathrm{Oe}\right)$, the fit deviates from the experimental data, while the fit considering only the high-field data extrapolates to $H_{C}=0$. At $H_{B}^{+}$, the experimental $H_{C}$ is still about $18 \%$ of its saturation value, approaching zero for $H_{\max }=0$ [Fig. 5(a), inset]. The differences between the $H_{\mathrm{CZFC}}$ and $H_{\mathrm{CFC}}$ data are probably of the order of data error and, thus, the differences between ZFC and FC fitted parameters are also within the error bars.

The value of $H_{C}$ at $H_{\max }=5 \times 10^{4}$ Oe and 3.2 and $4.2 \mathrm{~K}$ is of the order of that previously found for ferritin at $5 \mathrm{~K}(\sim 1700 \mathrm{Oe}){ }^{4}$ The slightly higher value that was found (2200 Oe) is probably due to the lower temperature. Other factors affecting $H_{C}$ that may contribute to this difference are the field-sweeping rate and characteristic time of measurement.

As in the case of $H_{C}$, the dependence of $H_{S}$ with $H_{\max }$ [Fig. 5(b)] can be in fact described by Eq. (12), with the parameters obtained for $H_{0}^{+}$and a constant $H_{0}^{-}=-0.297 \times$ $10^{4}$ Oe, which gives an extrapolated $H_{S}(\infty)=335$ Oe.

\section{CONCLUSIONS}

Coercivity and loop shifts in nanoparticles are dynamical phenomena, which depend on temperature, characteristic time of measurement, and number of cycles, for instance. In ferritin, we have shown that coercivity and loop shifts depend also on the cooling field and on the maximum field used for fields higher than those normally used. The dependence of coercivity and loop shifts with the maximum field can be described in terms of changes in the anisotropy energy barrier near zero field induced by the maximum field, and quantitatively described by a modified Néel-Brown model proposed here. Qualitatively, field cooling imprints energy barriers, such that the energy barriers near zero in the descending and ascending branches of the magnetization cycle are higher and lower than in the ZFC case, respectively. This difference is attenuated (but not erased) by increasing the maximum field in the opposite direction of the cooling field. Accordingly, the loop shift decreases with the maximum field, but it is not zero up to the highest field used $\left(30 \times 10^{4} \mathrm{Oe}\right)$, showing that the barriers imprinted by field cooling can not be overcome by these high fields.

The experimental observations and subsequent analysis presented in this paper have evidenced the imprinting of highenergy barriers through an effective anisotropy induced by the applied protocols. This gives rise to the high irreversibility and minor loop effects similar to those observed in spin glasses and diluted antiferromagnets, where this phenomenology is 
ascribed to dilution and the antiferromagnetic character of the interactions and not to frustration. ${ }^{31,32}$

\section{ACKNOWLEDGMENTS}

The authors acknowledge V. de Zea Bermudez for the synthesis of the organic-inorganic hybrid containing ferrihydrite nanoparticles and R. Boursier for his help with the high-field setup in Grenoble. Part of this work has been supported by EuroMagNET II under the EU Contract No. 228043. We acknowledge IFIMUP for the possibility of performing the magnetization time dependence measurements. The financial support from FCT (Grant No. PTDC/FIS/105416/2008) is gratefully recognized. The Aveiro-Zaragoza collaboration has been supported by the Integrated Spanish-Portuguese Action under the Grant No. PT2009-0131. The AveiroBarcelona collaboration has been supported by the Integrated Spanish-Portuguese Action under the Grant No. AIB2010PT00099. The work in Zaragoza has been supported by the research Grants No. MAT2007-61621 and No. CONSOLIDER CSD2007-00010 from the Ministry of Education. Oे.I. and A.L. acknowledge funding of the Spanish MICINN through Grants No. MAT2009-08667 and No. CSD2006-00012, and Catalan DIUE through Project No. 2009SGR856. N.J.O.S. acknowledges CSIC for a I3P contract and FCT for Ciencia 2008 program, A.U. acknowledges the financial support provided by contract from the EC NoE MAGMANET, and R.B. acknowledges ICMA-CSIC for a JAE-predoc grant. *nunojoao@ua.pt

${ }^{1}$ J. R. Friedman, U. Voskoboynik, and M. P. Sarachik, Phys. Rev. B 56, 10793 (1997).

${ }^{2}$ J. Tejada, X. X. Zhang, E. del Barco, J. M. Hernández, and E. M. Chudnovsky, Phys. Rev. Lett. 79, 1754 (1997).

${ }^{3}$ H. Mamiya, I. Nakatani, and T. Furubayashi, Phys. Rev. Lett. 88, 067202 (2002).

${ }^{4}$ S. A. Makhlouf, F. T. Parker, and A. E. Berkowitz, Phys. Rev. B 55, R14717 (1997).

${ }^{5}$ N. J. O. Silva, A. Millán, F. Palacio, E. Kampert, U. Zeitler, H. Rakoto, and V. S. Amaral, Phys. Rev. B 79, 104405 (2009).

${ }^{6}$ S. Mann, Inorganic Materials (Wiley, Chichester, 1996).

${ }^{7}$ N. J. O. Silva, V. S. Amaral, and L. D. Carlos, Phys. Rev. B 71, 184408 (2005).

${ }^{8}$ J. Nogués, J. Sort, V. Langlais, V. Skumryev, S. Suriñach, J. S. Muñoz, and M. D. Baró, Phys. Rep. 422, 65 (2005).

${ }^{9}$ Ò. Iglesias, A. Labarta, and X. Batlle, J. Nanosci. Nanotechnol. 8, 2761 (2008).

${ }^{10}$ S. Bendata, S. Sahoo, X. Chen, W. Kleemann, D. Sudfeld, K. Wojczykowski, and A. Hutten, Phase Transitions 78, 121 (2005).

${ }^{11}$ L. Néel, Ann. Géophys. 5, 99 (1949).

${ }^{12}$ W. F. Brown Jr., Phys. Rev. 130, 1677 (1963).

${ }^{13}$ E. C. Stoner and E. P. Wohlfarth, Philos. Trans. R. Soc., A 240, 599 (1948).

${ }^{14}$ N. J. O. Silva, V. S. Amaral, L. D. Carlos, B. Rodríguez-González, L. M. Liz-Marzán, T. S. Berquó, S. K. Banerjee, V. de Zea Bermudez, A. Millán, and F. Palacio, Phys. Rev. B 77, 134426 (2008).

${ }^{15}$ J. García-Otero, A. J. García-Bastida, and J. Rivas, J. Magn. Magn. Mater. 189, 377 (1998).

${ }^{16}$ H. Pfeiffer, Phys. Status Solidi A 118, 295 (1990).
${ }^{17}$ W. C. Nunes, M. A. Novak, M. Knobel, and A. Hernando, J. Magn. Magn. Mater. 226-230, 1856 (2001).

${ }^{18}$ C. P. Bean and J. D. Livingston, J. Appl. Phys. 30, S120 (1959).

${ }^{19}$ E. F. Kneller and F. E. Luborsky, J. Appl. Phys. 34, 656 (1963).

${ }^{20}$ L. He and C. Chen, Phys. Rev. B 75, 184424 (2007).

${ }^{21}$ N. J. O. Silva, V. S. Amaral, L. D. Carlos, and V. de Zea Bermudez, J. Appl. Phys. 93, 6978 (2003)

${ }^{22}$ N. J. O. Silva, V. S. Amaral, L. D. Carlos, B. Rodríguez-González, L. M. Liz-Marzán, A. Millán, F. Palacio, and V. de Zea Bermudez, J. Appl. Phys. 100, 054301 (2006).

${ }^{23}$ S. H. Kilcoyne and R. Cywinski, J. Magn. Magn. Mater. 140-144, 1466 (1995).

${ }^{24}$ A. Punnoose, T. Phanthavady, M. S. Seehra, N. Shah, and G. P. Huffman, Phys. Rev. B 69, 054425 (2004).

${ }^{25}$ A. E. Berkowitz, R. Kodama, S. A. Makhlouf, F. T. Parker, F. E. Spada, E. J. McNiff Jr., and S. Foner, J. Magn. Magn. Mater. 196-197, 591 (1999).

${ }^{26}$ H. Kachkachi and H. Mahboub, J. Magn. Magn. Mater. 278, 334 (2004).

${ }^{27}$ T. G. S. Pierre, N. T. Gorham, P. D. Allen, J. L. Costa-Krämer, and K. V. Rao, Phys. Rev. B 65, 024436 (2001).

${ }^{28}$ T. Jonsson, J. Mattsson, P. Nordblad, and P. Svedlindh, J. Magn. Magn. Mater. 168, 269 (1997).

${ }^{29}$ F. Luis, E. del Barco, J. M. Hernández, E. Remiro, J. Bartolomé, and J. Tejada, Phys. Rev. B 59, 11837 (1999).

${ }^{30}$ H. Mamiya, M. Ohnuma, I. Nakatani, and T. Furubayashi, Europhys. Lett. 74, 500 (2006).

${ }^{31}$ P. M. Levy, C. Morgan-Pond, and A. Fert, J. Appl. Phys. 53, 2168 (1982).

${ }^{32}$ R. Rodríguez, A. Fernández, A. Isalgué, J. Rodríguez, A. Labarta, J. Tejada, and X. Obradors, J. Phys. C: Solid State Phys. 18, L401 (1985). 